\title{
Editorial
}

\section{Nonlinear Problems: Mathematical Modeling, Analyzing, and Computing for Finance}

\author{
Chuangxia Huang, ${ }^{1}$ Fenghua Wen, ${ }^{2}$ Jianping $\mathrm{Li}^{3}{ }^{3}$ and Xiaodong $\mathrm{Lin}^{4}$ \\ ${ }^{1}$ College of Mathematics and Computing Science, Changsha University of Science and Technology, Changsha 410114, China \\ ${ }^{2}$ School of Business, Central South University, Changsha, Hunan 410083, China \\ ${ }^{3}$ Institute of Policy and Management, Chinese Academy of Sciences (CAS), Beijing 100190, China \\ ${ }^{4}$ Rutgers Business School, Rutgers University, Piscataway, NJ 08854, USA
}

Correspondence should be addressed to Chuangxia Huang; cxiahuang@126.com

Received 1 June 2014; Accepted 1 June 2014; Published 18 June 2014

Copyright (C) 2014 Chuangxia Huang et al. This is an open access article distributed under the Creative Commons Attribution License, which permits unrestricted use, distribution, and reproduction in any medium, provided the original work is properly cited.

Modern financial markets encapsulate vast number of interconnected financial entities, instruments, and strategies. Understanding these complex dynamical systems requires multidisciplinary efforts from a wide range of quantitative fields including mathematics, statistics, data mining, and operations research. While conventional financial research focuses mostly on linear models of variables of interest, they cannot cope with real-world financial phenomena. In the past decade, we have seen significant progress in our fundamental understanding of dynamical financial and economic behaviors, both from the micro- and macroprospectives, using nonlinear systems and methodologies. Powerful techniques borrowed from traditional nonlinear models and new methods invented have been brought to almost every aspect of financial research, including asset pricing, risk management, and financial forecasting. Still, there exist many challenging problems. In spite of the amount of published results recently focused on dynamical financial and economic behaviors, there remain many challenging open questions.

The aim of this special issue is to gather recent research efforts on the development and applications of nonlinear techniques to address the critical issues in finance and to see the latest developments. The original papers explored in this special issue include a wide variety of topics such as the following.

Financial Time Series Modeling and Forecasting. Y. Zhang et al. proposed a hybrid system to predict corporate bankruptcy.
E. Momoniat et al. used the backward difference formulae to improve the prediction of direction in market related data. K. He et al. proposed entropy optimized multivariate wavelet denoising model to forecast exchange rate. Q. Zhu et al. developed the RBF-QRNN model and BVAR model separately to predict future gas consumption.

Risk Assessment and Credit Analysis. R. Chen et al. proposed a method for measuring the stock market's risk and earlywarning methods which are based on price-to-earnings ratio and price-to-book ratio. Y. Cao and S. Xiong built a sustainable financing credit rating index system for smalland medium-sized enterprises in China from the perspective of sustainability. How do prior outcomes affect the risk choice? F. Wen et al. found that the prior gains and losses have an impact on the form of value function and the current investors' risk attitude. S. Du et al. studied the impact of emergencies on the supplier's decision-making behaviors including production and information sharing in consideration of consumer risk perception, consumer loss aversion phenomenon, and government price control. G. Kou and $\mathrm{W}$. Wu proposed an analytic hierarchy model (AHM) to evaluate classification algorithms for credit risk analysis. W.-G. Zhang and P.-K. Liao investigated the convertible bonds pricing problem with regime switching and credit risk in the convertible bond market. Z. Xiaoqin and C. Huang developed a martingale approach to investigate two mixed claim frequency risk models. 
Asset Pricing and Arbitrage Techniques. J. Liu et al. focused on the pricing problem of European options and convertible bonds by using an actuarial approach and established the pricing formula of European options. H. Xiaoping and C. Jie showed that the impact of the occurrence probability of the random binomial tree environment on American option prices is very significant. J. Sheng et al. found that the market beta and the benchmark beta that is related to the investor's regret are the determinants of equilibrium asset prices. W. Bi et al. studied a dynamic pricing model dealing with repeated decision problems in a duopoly market. L. Ping and W. Xiaoxu extended incomplete information model by combining with the credit risk premium and redefined the pricing formula for defaultable corporate bonds.

Portfolio Selection and Optimization. H. Ding et al. investigated the theoretical foundation of DEA (data envelopment analysis) approach to evaluate the performance of portfolios with margin requirements from a different perspective. $\mathrm{M}$. Wang et al. proposed a new auction mechanism which converges to a unique Pareto optimal equilibrium in a finite number of periods. A. Ling and L. Tang considered a numerical study of a robust active portfolio selection model with downside risk and multiple weights constraints. D. Zhao and Y. Fang showed that the representation bias information is useful to the return forecast as well as the portfolio selection.

Nonlinear Dynamical System for Finance. Z. Ouyang and H. Wang established a model for influence of nuclear-electricity industry on area economy and constructed the approximation conditions such that the economic aggregate will tend to the equilibriums point as time tends to infinity. Y. Hu et al. suggested a generalized method of moments- (GMM-) based estimation for dynamic panel data models with individual specific fixed effects and threshold effects simultaneously. J. Xiao et al. proposed a one-step dynamic classifier ensemble model for missing values (ODCEM) model. G. Zhang et al. used input-output method to make a contrastive analysis of changes in carbon emissions caused by Chinese rural and urban residents' consumption. W. Chen et al. applied Markov chain approach to diagnose the convergence of financial agglomeration in China based on the location quotient coefficients across the provincial regions over 19932011. K. Shi et al. were concerned with the problem of delaydependent robust stability analysis for a class of uncertain neutral type Lur'e systems with mixed time-varying delays.

Behavioral Finance Modeling. X. Zhang et al. presented a three-level oligopoly power producer's capacity investment game model. H. Zhang and Q. Lan proposed a new volatility model which is called as GARCH-type model with continuous and jump variation (GARCH-CJ-type model) for the research on the capital assets pricing. $Z$. Liu et al. investigated the modern social interaction effects on the stock market participation and its activeness. Y. Yue and J. Zou captured the correlation between the choices of health insurance and pension insurance using the bivariate probit model and studied the effect of wealth and health on insurance choice.
J. Huang et al. proposed a two-stage network model with bad outputs and supper efficiency (US-NSBM) to open the black box of input-output production of banks and provide comprehensive and accurate assessment on the efficiency of each stage. C. Huang et al. constructed a proxy variable to examine the relationship between investor sentiment and the return of a specific industry. $H$. Che et al. found that the future contracts generally have a larger number of information transactions.

Energy and Emissions Markets. G.-J. Wang et al. supplied a new perspective to describe and understand the behavior of cross-correlations between energy and emissions markets. They analyzed short-term and long-term market dynamics and found that the recent global financial crisis has a notable influence on short-term and long-term market dynamics. Q. Dai et al. proposed an approach based on the proportional allocation concept to allocate TEPs among a set of decisionmaking units (DMUs) and showed that the developed allocation method can be helpful for achieving a saving in energy consumption and reducing emissions.

In a word, the response to this special issue was beyond our expectation. We received 114 papers in the interdisciplinary research fields. This special issue includes 45 highquality peer-reviewed articles. These articles contain several new, novel, and innovative techniques and ideas that may stimulate further research in every branch of pure and applied sciences.

\section{Acknowledgments}

The authors would like to express their deepest gratitude to the reviewers, whose professional comments and valuable suggestions guaranteed the high quality of these selected papers. The editors would like to express their gratitude to the authors for their interesting and novel contributions. The interested readers are advised to explore these interesting and fascinating fields further. The authors hope that problems discussed and investigated in this special issue may inspire and motivate discovering new, innovative, and novel applications in all areas of financial engineering.

Chuangxia Huang
Fenghua Wen
Jianping Li
Xiaodong Lin




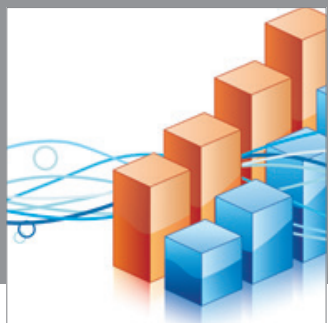

Advances in

Operations Research

mansans

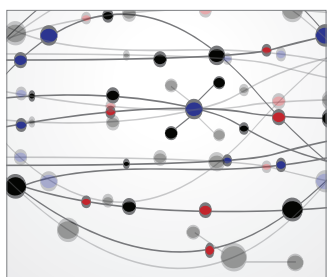

The Scientific World Journal
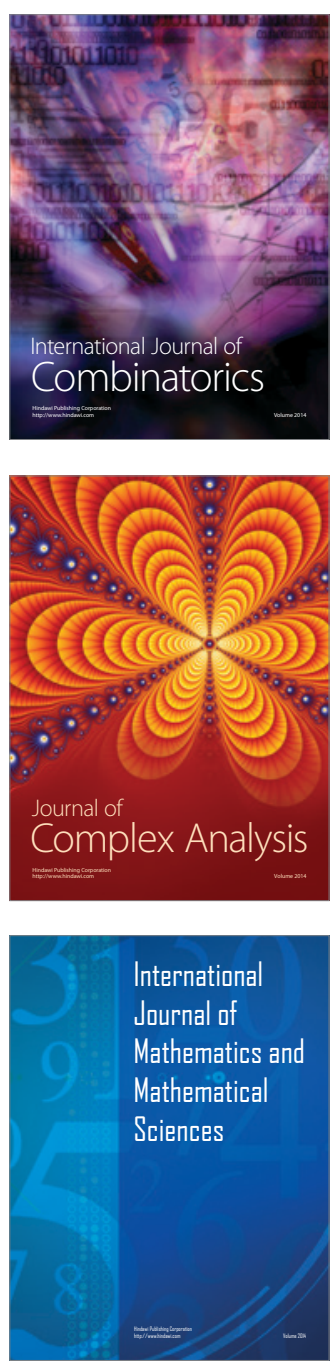
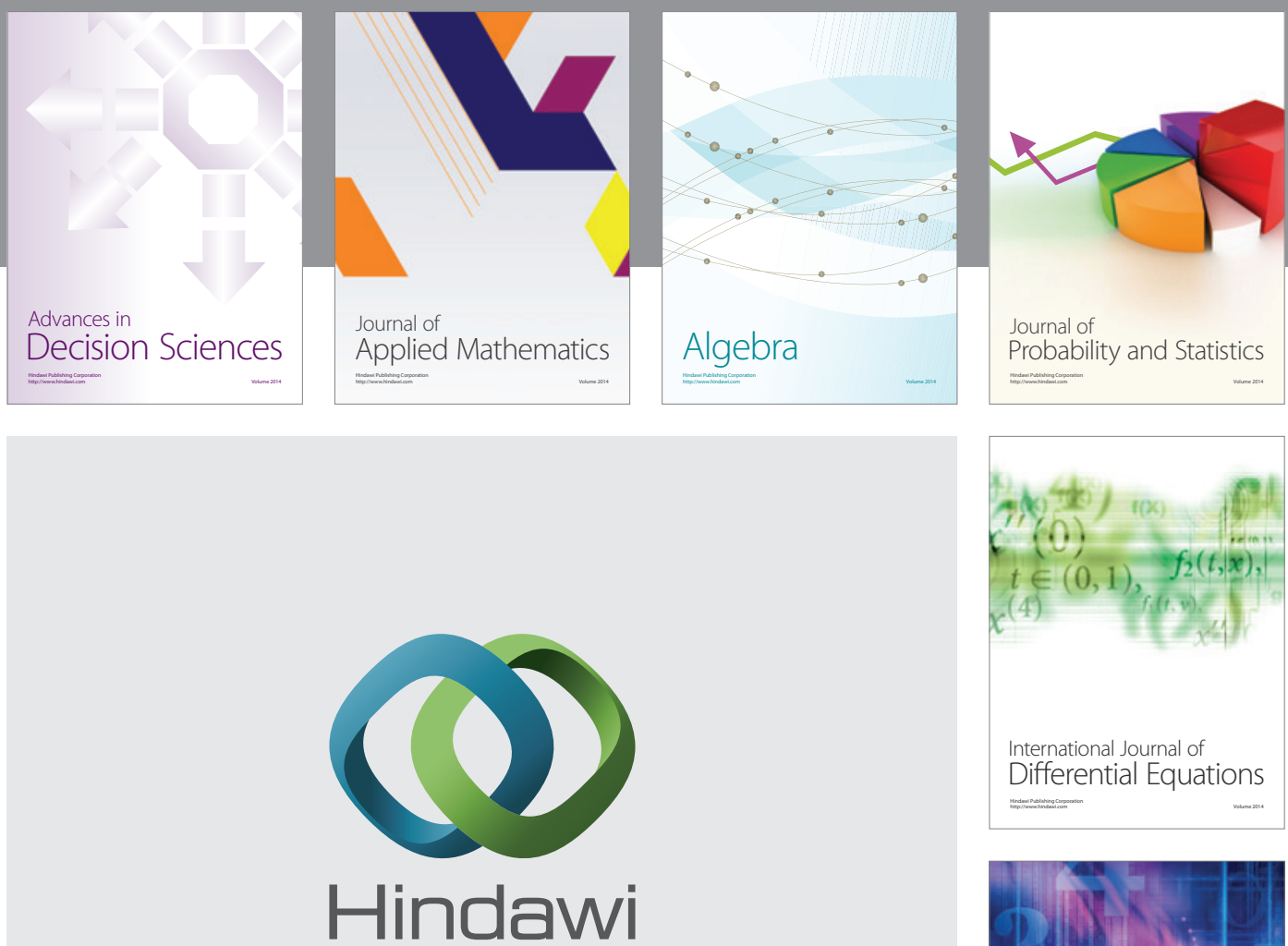

Submit your manuscripts at http://www.hindawi.com
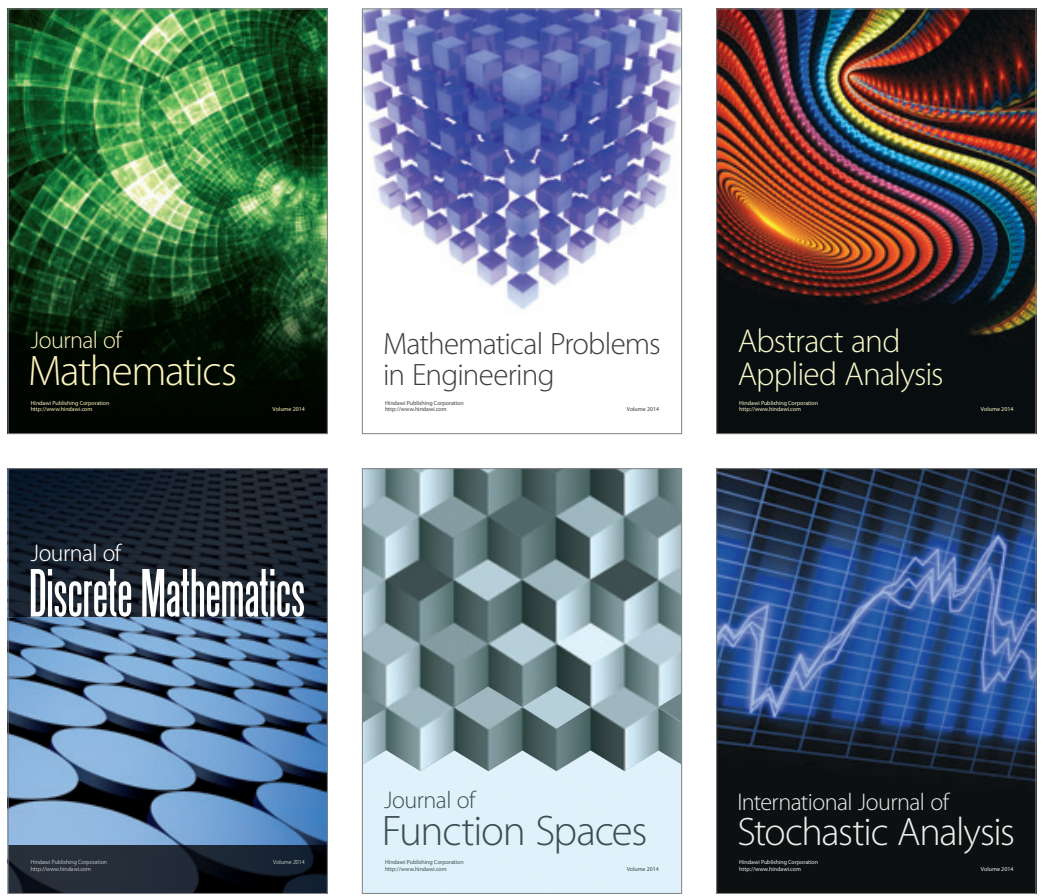

Journal of

Function Spaces

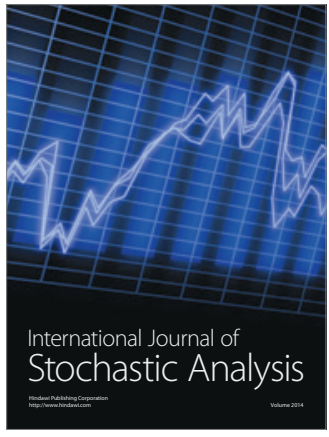

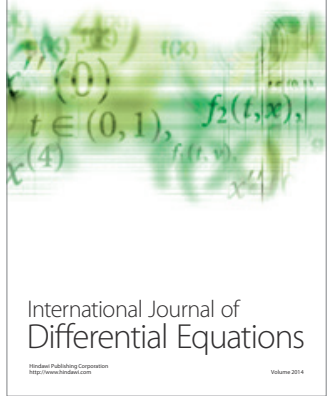
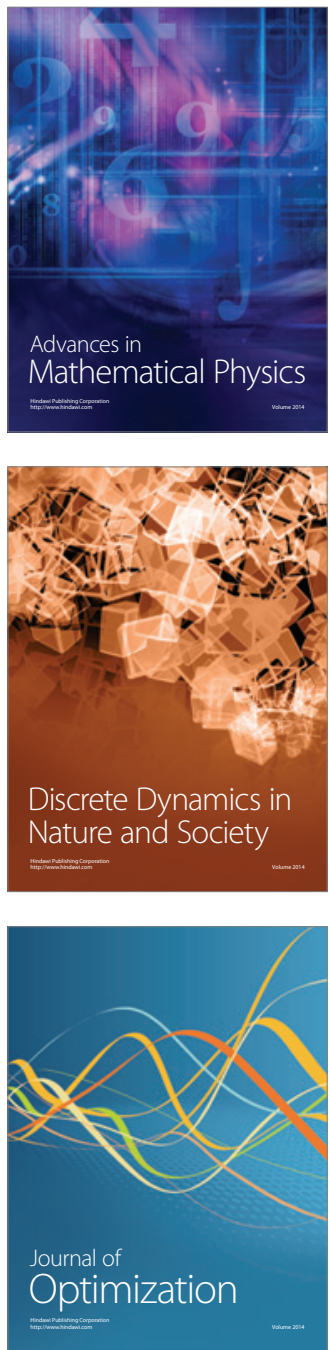